a. Laboratorio de Investigaciones en Adaptación y Ontogenia (LINOA), Facultad de Ciencias Naturales y Museo, Universidad Nacional de La Plata (UNLP).

b. Instituto de Genética Veterinaria (IGEVET) Facultad de Ciencias Veterinarias (FCV)Universidad Nacional de La Plata (UNLP)-Centro La Plata (UNLP)-Centro Cientifico Tecnologico (CCT), Consejo Nacional de Investigaciones Científic y Técnicas (CONICET). La Plata, Buenos Aires. Instituto de Ciencias Antropológicas (ICA), Facultad de Filosofía y Letras, Universidad de Buenos Aires, Argentina.

d. Instituto de Ecorregiones Andinas (INECOA), Universidad Nacional de Jujuy (UNJu)-CONICET. Instituto de Biología de la Altura, UNJu.

f. Unidad de Investigaciones en Antropología Biológica (UIAB), Facultad de Humanidades y Ciencias Sociales, UNJu.

Laboratorio de Antropología Biológica, y Evolución Austral y Evolución Austral (IDEAUS), CCT-Centro Nacional Patagónico (CENPAT)-CONICET.

h. Facultad de Ciencias Naturales y de la Salud, Universidad Nacional San Juan Bosco (UNPSJB).

Centro de Estudios de Antropología Biológica (CEABI), Universidad Nacional de Catamarca (UNCA).

Instituto Regional de Estudios Socioculturales (IRES), CONICET-UNCA

k. Centro de Estudios Parasitológicos y de Vectores (CEPAVE), UNLP-CCT, CONICET. Argentina.

Arupo de Investigación de Epidemiología de la Nutrición (EPINUT) Departamento de Biodiversidad, Ecología y Evolución, Facultad de Ciencias Biológicas, Universidad Complutens de Madrid, España.

Correspondencia:

Dra. Evelia E. Oyhenart: oyhenart@fcnym.unlp.edu.ar.

Financiamiento:

Los fondos correspondieron a los aportados en Argentina por la Agencia Nacional de Promoción Científica y Tecnológica (ANPCyT: PICT 01541; PICT 07391; PICTO 00139; PICTO 32451), el Consejo Nacional de Investigacione Científicas y Técnicas (CONICET: PIP 0106) y la Secretaría de Ciencia y Técnica (SECyT: UNLP 11N/679; UNCA L449; UNJu F0013; UNPSJB C30), y los de España correspondieron al Ministerio de Educación y Ciencia de España (05372)

Conflicto de intereses: Ninguno que declarar.

Recibido: 2-9-2018 Aceptado: 31-1-2019

\title{
Percentilos de referencia de la circunferencia y de las áreas muscular y grasa del brazo para la población infantojuvenil argentina (4-14 años) \\ Reference percentiles for mid-upper arm circumference, upper arm muscle and fat areas in the Argentine child and adolescent population (4-14 years old)
}

\author{
Dra. Evelia E. Oyhenart ${ }^{a, b}$, Dra. María F. Torres ${ }^{b, c}$, Dra. Mariela Garraza $a^{a, b}$, \\ Dra. María F. Cesani $i^{a, b}$, Lic. Bárbara Navazo ${ }^{a, b}$, Dr. Luis E. Castro ${ }^{a}$, \\ Dra. Emma Alfaro ${ }^{d, e}$, Mg. Ignacio F. Bejarano e,f, Lic. Rafael Carrilloef, \\ Dra. Silvia L. Dahinten ${ }^{g, h}$, Dra. Delia Lomaglio ${ }^{i, j}$, Lic. María A. Luis ${ }^{a}$, \\ Lic. Natalia Menecier ${ }^{i}$ Dr. Fabián A. Quintera ${ }^{a, b}$, Lic. Estela M. Román ${ }^{d, e}$, \\ Dra. María L. Zontak ${ }^{k}$ Dra. María D. Marrodán Serrano y Dr. José E. Dipierrid,e
}

\section{RESUMEN}

Introducción: La circunferencia del brazo (CB) se reconoce como buen indicador del estado nutricional.

Objetivo: Estimar los percentilos de referencia para la CB y las áreas muscular (AM) y grasa (AG) del brazo en la población infantojuvenil argentina mediante el empleo del método LMS (lambda, mu, sigma, en inglés).

Materiales y métodos: La muestra estuvo constituida por escolares de 4,0 a 13,9 años residentes en Jujuy, Catamarca, Misiones, Buenos Aires, Mendoza y Chubut. Las mediciones antropométricas dela CBy del pliegue tricipital se realizaron entre2003 y 2008 siguiendo protocolos estandarizados. Se estimaron las AM, AG, se calcularon los percentilos por edad y sexo, y se compararon mediante el análisis de la varianza. Resultados: Se incluyeron 22736 escolares (11397 varones y 11339 mujeres). Los valores correspondientes al percentilo 50 fueron superiores, en las mujeres, para CB y AG, y, en los varones, para $\mathrm{AM}$. Las curvas de $\mathrm{CB}$ presentaron incrementos más marcados a partir de los 7 años en todos los percentilos, para ambos sexos. Un patrón similar se observó para AM, con valores superiores en los varones. Por último, el AG mostró aumento constante en las mujeres y estabilización en los varones a partir de los 11 años. Se observaron diferencias para la edad. Conclusiones: Los valores de percentilos, tabulados y graficados, de la CB y de las AMy AG del brazo pueden constituir una referencia local para estudios epidemiológicosy antropológicos. Palabras clave:valores de referencia, antropometría, composición corporal, Argentina, método LMS.

http:/ / dx.doi.org/10.5546/aap.2019.e347

Texto completo en inglés:

http:/ / dx.doi.org/10.5546/ aap.2019.eng.e347

Cómo citar: Oyhenart EE, Torres MF, Garraza M, Cesani MF, et al. Percentilos de referencia de la circunferencia y de las áreas muscular y grasa del brazo para la población infantojuvenil argentina (414 años). Arch Argent Pediatr 2019;117(4):e347-e355.

\section{INTRODUCCIÓN}

Las mediciones antropométricas constituyen herramientas valiosas para la evaluación del crecimiento y del estado nutricional, dada su simplicidad metodológica y bajo costo en estudios a gran escala. ${ }^{1}$ En este sentido, con fines comparativos, es importante contar con valores de referencia que reflejen, en lo posible, la variabilidad presente en la población.

Evaluaciones auxológicas previas, efectuadas en grupos poblacionales de Argentina, han demostrado la presencia de heterogeneidad en sus patrones de crecimiento. Esta diversidad se atribuye, fundamentalmente, al efecto de factores ambientales, tales como los geoclimáticos, económicos, sociales y culturales, que caracterizan el extenso territorio nacional. ${ }^{2-5}$

Desde 1920, se ha reconocido que la circunferencia del brazo (CB) es un indicador del estado nutricional de poblaciones en diversos lugares del mundo. ${ }^{6}$ Esta medida antropométrica es particularmente útil en situaciones de emergencia en las que se requiere una evaluación rápida en terreno, ${ }^{7,8}$ en lugares aislados donde no se cuenta con estadiómetros, balanzas $\mathrm{u}$ otros instrumentos de medición, ${ }^{9,10}$ durante situaciones de hambruna o crisis de refugiados en las que es difícil determinar la estatura y el peso, especialmente, de niños desnutridos, ${ }^{11}$ 
o cuando la medición del peso corporal se considera inapropiada por inducir problemas de imagen asociados a un modelo ideal. ${ }^{12}$ Asimismo, la $\mathrm{CB}$ se ha usado como instrumento adicional de tamizaje y preventivo, dado su poder para predecir la mortalidad infantil ${ }^{13}$ por tratarse de una medición confiable, con bajo error entre observadores y fácilmente registrable por los agentes de salud.

Estudios recientes demuestran que, además de la aplicación tradicional de la CB en la estimación de la desnutrición, su empleo resulta de gran utilidad para la evaluación clínica y la vigilancia epidemiológica de la obesidad en países en desarrollo. ${ }^{9,10,14}$ La combinación de la CB con el pliegue subcutáneo tricipital (PT) permite calcular, indirectamente, las áreas grasa (AG) y magra del brazo. ${ }^{7}$ La estimación de estos parámetros de composición corporal posibilita no solo conocer las reservas calóricas y proteicas, sino también identificar los factores de riesgo relacionados con la malnutrición, tanto por déficit como por exceso. ${ }^{15}$ La fiabilidad de los cálculos derivados de la antropometría del brazo fue validada con los estudios de resonancia magnética hace, aproximadamente, dos décadas y se demostró alta correlación, especialmente, del AG. ${ }^{16}$

Desde 1976, la National Health Survey ha publicado, junto con otras medidas antropométricas, los valores de los percentilos de la CB para los varones y las mujeres de la población de Estados Unidos, con edades comprendidas entre los 2 meses y los 19 años. ${ }^{17}$ Sin embargo, mientras que la World Health Organization (WHO), en un primer momento, no había recomendado ni incluido en las tablas de los niños de 5 a 19 años la $C B,{ }^{13,18}$ esta fue considerada entre las mediciones del Multicentre Growth Reference Study Group para los niños de 0 a 5 años. ${ }^{19}$

Para el análisis de la composición corporal mesobraquial, realizado mediante la $C B$ y las variables derivadas áreas muscular (AM) y AG, existen referencias muy utilizadas, como la de Frisancho ${ }^{20}$ y la más recientemente publicada por Addo et al., ${ }^{21}$ basadas en encuestas nacionales de salud de la población estadounidense. En Argentina, solo se cuenta, hasta el momento, con valores de referencia de la CB para poblaciones de Córdoba ${ }^{22}$ y Buenos Aires. ${ }^{8}$

En este contexto, considerando los aspectos anteriormente señalados, el presente estudio tuvo por objetivo estimar los percentilos de referencia de la CB y las AM y AG del brazo para la población infantojuvenil argentina.

\section{POBLACIÓN Y MÉTODOS Población}

La muestra estuvo constituida por escolares, de los niveles inicial, primario y secundario, residentes en seis provincias de las cinco regiones geográficas del país: Jujuy y Catamarca (Noroeste), Misiones (Noreste), Buenos Aires (Centro), Mendoza (Cuyo) y Chubut (Patagonia). El muestreo fue de carácter no probabilístico y por conveniencia. De cada provincia fueron incluidas las siguientes ciudades y localidades: San Salvador de Jujuy, Susques, Fraile Pintado y Humahuaca (Jujuy); San Fernando del Valle de Catamarca y El Peñón (Catamarca); Aristóbulo del Valle (Misiones); Brandsen, La Plata, Magdalena y Punta Indio (Buenos Aires); General Alvear y San Rafael (Mendoza); Puerto Madryn (Chubut). La autorización para el ingreso a los establecimientos educativos fue tramitada ante las autoridades escolares de cada distrito. La población elegible estuvo constituida por todos los escolares de 4,0 a 13,9 años de edad que estuvieron presentes el día del relevamiento antropométrico y que contaron con la autorización escrita de los padres/ madres/tutores. Fueron excluidos los niños con enfermedad crónica o aguda, con indicación medicamentosa al momento del estudio, sin autorización escrita de los padres/madres / tutores y los que, aun teniéndola, no quisieron participar.

\section{MÉTODOS}

El diseño del estudio fue descriptivo transversal. Las mediciones fueron realizadas en establecimientos educativos públicos durante los ciclos escolares de los años 2003-2008, siguiendo protocolos estandarizados por la International Society for the Advancement of Kinanthropometry (ISAK). ${ }^{23}$ Fueron relevados, sobre el lado izquierdo del brazo relajado, la $\mathrm{CB}$ en centímetros empleando una cinta métrica inextensible (precisión de $1 \mathrm{~mm}$ ) y el PT en milímetros utilizando el calibre Lange de presión constante (precisión de $1 \mathrm{~mm}$ ). El instrumental se calibró al comienzo de cada sesión antropométrica. Todas las mediciones antropométricas fueron relevadas por los autores, especialistas entrenados en técnicas antropométricas. Antes del relevamiento de los datos, todos los antropometristas se reunieron y se calculó el error intra- e interobservador, que fue 
menor del $5 \%$, lo que aseguró la estandarización de las medidas. ${ }^{23}$

A partir de las variables antropométricas relevadas, se estimó el área total (AT), el AM y el AG del brazo empleando las fórmulas propuestas por Frisancho: ${ }^{20}$

$\mathrm{AT}=\left[(\pi / 4) \times\left(\mathrm{CB} / \pi^{2}\right)\right]$

$\mathrm{AM}=(\mathrm{CB}-\pi \times \mathrm{PT})^{2} / 4 \pi$

$\mathrm{AG}=\mathrm{AT}-\mathrm{AM}$

Se calculó la edad decimal de cada niño a partir de la fecha de nacimiento obtenida del documento nacional de identidad o de los registros escolares y de la fecha de medición.

\section{Análisis estadístico}

Los datos se agruparon por sexo y edad decimal. Cada año decimal fue dividido en dos, por lo que se conformaron 21 rangos etarios. Se analizó la dispersión de los datos crudos y se suprimieron las mediciones extremas utilizando como punto de corte \pm 4 desvíos estándar (DE). ${ }^{2}$ Con este criterio, se eliminaron 42 casos del total (el 0,18\%).

Para el cálculo de los valores de los percentilos, se utilizó el método LMS. Este sintetiza la distribución cambiante de las mediciones antropométricas en función de la edad mediante las curvas lambda (L), mu (M) y sigma (S), que representan, respectivamente, la asimetría, la mediana y la varianza. Utiliza la transformación Box-Cox (L) para adecuar la distribución de los datos antropométricos a una distribución normal, minimizando, fundamentalmente, los efectos de la asimetría, pero no de la curtosis. ${ }^{24}$ Los parámetros L, M y S se calcularon de acuerdo con el procedimiento de la máxima probabilidad penalizada. ${ }^{24}$

El cálculo de los percentilos $(\mathrm{P})$ se realizó de acuerdo con la siguiente fórmula: ${ }^{25}$

$\mathrm{P}=\mathrm{M}[1+\mathrm{LSZ}]^{1 / \mathrm{L}}$

En esta, L, M y $S$ eran los valores calculados para cada edad y $\mathrm{Z}$ era el puntaje $\mathrm{z}(\mathrm{Pz})$ correspondiente al percentilo buscado. Para estimar la bondad de ajuste, se utilizó el test Q. Se empleó la edad decimal de los escolares, y se configuraron las curvas para que el eje Y mostrara las edades en números enteros y los puntos equidistantes entre enteros.

El procesamiento de los datos se realizó empleando el programa LMS ChartMaker Pro. Dicha versión permitía ajustar los modelos teniendo en cuenta el peso de las muestras y la selección de grupos. Asimismo, posibilitaba evaluar la bondad del ajuste mediante la prueba Q. Los grados de libertad de L, M y S que se utilizaron para el ajuste de la curva correspondieron, respectivamente, a 3-5-3. En tanto que, para graficar las curvas, se usó el programa R 3.2.0.

Los percentilos calculados por edad para $\mathrm{CB}$, AM y AG, en cada sexo, se compararon gráficamente y se analizaron mediante el análisis de la varianza (analysis of variance; ANOVA, por sus siglas en inglés).

\section{Consideraciones éticas}

Las investigaciones se ajustaron a lo establecido por la Declaración Universal de los Derechos Humanos de 1948, las normas éticas instituidas por el Código de Nüremberg de 1947 y la Declaración de Helsinki de 1964 y sucesivas enmiendas, y se atendió, especialmente, a lo normado por la Ley Nacional 25326 de protección de datos personales.

El estudio fue aprobado por el Comité del Hospital de la Escuela Interzonal "San Juan Bautista" de Catamarca, el Comité de Bioética de la provincia de Jujuy y el Comité de Bioética de la Escuela Latinoamericana de Bioética (CELABE) para las provincias de Buenos Aires, Chubut, Mendoza y Misiones.

\section{RESULTADOS}

El estudio incluyó a 22736 niños (11397 varones y 11339 mujeres) de 4,0 a 13,9 años de edad. Correspondieron a Jujuy 1700 (842 varones y 858 mujeres); a Catamarca, 941 (456 varones y 485 mujeres); a Misiones, 2206 (1088 varones y 1118 mujeres); a Buenos Aires, 8420 (4177 varones y 4243 mujeres); a Mendoza, 6652 (3369 varones y 3283 mujeres) y, a Chubut, 2817 (1465 varones y 1352 mujeres).

La muestra tuvo la siguiente representación por edad y sexo: 4,0-4,9 años (1238: 637 varones, 601 mujeres); 5,0-5,9 años (1940: 1015 varones, 925 mujeres); 6,0-6,9 años (2405: 1253 varones, 1152 mujeres); 7,0-7,9 años (2510: 1292 varones, 1218 mujeres); 8,0-8,9 años (2649: 1284 varones, 1365 mujeres); 9,0-9,9 años (2720: 1375 varones, 1345 mujeres); 10,0-10,9 años (2631: 1275 varones, 1356 mujeres); 11,0-11,9 años (2593: 1276 varones, 1317 mujeres); 12,0-12,9 años (2319: 1117 varones, 1202 mujeres) y 13,0-13,9 años (1731: 873 varones, 858 mujeres).

En las Tablas 1-3 y en las Figuras 1-3, se presentan los valores en el formato de nueve percentilos: 3, 5, 10, 25, 50, 75, 90, 95 y 97, correspondientes a CB, AM y AG para los 
varones y las mujeres de 4 a 14 años, clasificados por períodos de 6 meses. Las tablas también muestran los parámetros L y S.

En general, los valores correspondientes al P50 fueron superiores, en las mujeres, para $\mathrm{CB}$ y $\mathrm{AG}$, $\mathrm{y}$, en los varones, para AM (Tablas 1-3).

Las curvas de CB presentaron incrementos que fueron más marcados a partir de los 7 años en todos los percentilos, para ambos sexos (Figura 1). Un patrón similar se observó para el AM, con valores superiores en los varones (Figura 2). Por último, el AG mostró un aumento constante en las mujeres y la estabilización en los varones a partir de los 11 años (Figura 3).

Se observaron diferencias estadísticamente significativas en todas las comparaciones por edad. Para $C B$, varones: $F=2,515, p<0,0001$; mujeres: $\mathrm{F}=2,769, \mathrm{p}<0,0001$; para $\mathrm{AM}$, varones: $\mathrm{F}=3,405, \mathrm{p}<0,0001$; mujeres: $\mathrm{F}=3,485, \mathrm{p}<0,0001$; para $A G$, varones: $F=1,260, p<0,0001$; mujeres: $\mathrm{F}=1,425, \mathrm{p}<0,0001$.

TABla 1. Percentilos $(P)$ y valores de la asimetría $(L)$ y coeficiente de variación $(S)$ para la circunferencia del brazo en los varones y las mujeres

\begin{tabular}{|c|c|c|c|c|c|c|c|c|c|c|c|}
\hline$\underline{\text { Edad (años) }}$ & $\mathbf{L}$ & $\mathrm{S}$ & P3 & P5 & P10 & P25 & P50 & P75 & P90 & P95 & P97 \\
\hline \multicolumn{12}{|l|}{ Varones } \\
\hline 4,0 & $-2,4023$ & 0,0843 & 14,70 & 14,92 & 15,28 & 15,94 & 16,81 & 17,86 & 19,02 & 19,85 & 20,46 \\
\hline 4,5 & $-2,5126$ & 0,0864 & 14,83 & 15,05 & 15,41 & 16,09 & 16,99 & 18,08 & 19,31 & 20,20 & 20,87 \\
\hline 5,0 & $-2,6094$ & 0,0886 & 14,95 & 15,17 & 15,54 & 16,23 & 17,15 & 18,29 & 19,59 & 20,55 & 21,27 \\
\hline 5,5 & $-2,6723$ & 0,0909 & 15,03 & 15,26 & 15,63 & 16,34 & 17,29 & 18,47 & 19,84 & 20,87 & 21,65 \\
\hline 6,0 & $-2,6917$ & 0,0934 & 15,07 & 15,30 & 15,69 & 16,41 & 17,39 & 18,62 & 20,06 & 21,15 & 21,99 \\
\hline 6,5 & $-2,6721$ & 0,0963 & 15,12 & 15,36 & 15,75 & 16,50 & 17,51 & 18,79 & 20,30 & 21,45 & 22,35 \\
\hline 7,0 & $-2,6350$ & 0,0995 & 15,22 & 15,47 & 15,87 & 16,65 & 17,70 & 19,03 & 20,63 & 21,86 & 22,81 \\
\hline 7,5 & $-2,5920$ & 0,1031 & 15,38 & 15,63 & 16,05 & 16,86 & 17,96 & 19,37 & 21,07 & 22,38 & 23,42 \\
\hline 8,0 & $-2,5368$ & 0,1067 & 15,58 & 15,84 & 16,29 & 17,13 & 18,29 & 19,78 & 21,59 & 23,00 & 24,12 \\
\hline 8,5 & $-2,4538$ & 0,1106 & 15,82 & 16,10 & 16,56 & 17,45 & 18,67 & 20,26 & 22,18 & 23,69 & 24,89 \\
\hline 9,0 & $-2,3352$ & 0,1146 & 16,07 & 16,36 & 16,85 & 17,80 & 19,09 & 20,77 & 22,81 & 24,41 & 25,68 \\
\hline 9,5 & $-2,1857$ & 0,1187 & 16,30 & 16,62 & 17,13 & 18,14 & 19,51 & 21,28 & 23,43 & 25,11 & 26,44 \\
\hline 10,0 & $-2,0179$ & 0,1226 & 16,51 & 16,85 & 17,40 & 18,46 & 19,91 & 21,78 & 24,02 & 25,76 & 27,12 \\
\hline 10,5 & $-1,8499$ & 0,1259 & 16,72 & 17,07 & 17,66 & 18,78 & 20,30 & 22,25 & 24,57 & 26,35 & 27,72 \\
\hline 11,0 & $-1,6813$ & 0,1286 & 16,92 & 17,29 & 17,90 & 19,08 & 20,68 & 22,70 & 25,07 & 26,86 & 28,23 \\
\hline 11,5 & $-1,5160$ & 0,1307 & 17,10 & 17,49 & 18,14 & 19,38 & 21,04 & 23,12 & 25,52 & 27,31 & 28,65 \\
\hline 12,0 & $-1,3531$ & 0,1319 & 17,33 & 17,74 & 18,42 & 19,71 & 21,42 & 23,56 & 25,97 & 27,74 & 29,05 \\
\hline 12,5 & $-1,1754$ & 0,1325 & 17,59 & 18,02 & 18,73 & 20,07 & 21,85 & 24,02 & 26,43 & 28,16 & 29,42 \\
\hline 13,0 & $-0,9772$ & 0,1323 & 17,88 & 18,34 & 19,09 & 20,49 & 22,31 & 24,51 & 26,90 & 28,57 & 29,78 \\
\hline 13,5 & $-0,7580$ & 0,1315 & 18,21 & 18,69 & 19,48 & 20,94 & 22,82 & 25,03 & 27,37 & 28,98 & 30,12 \\
\hline 14,0 & $-0,5243$ & 0,1305 & 18,55 & 19,06 & 19,89 & 21,41 & 23,34 & 25,55 & 27,85 & 29,39 & 30,46 \\
\hline \multicolumn{12}{|l|}{ Mujeres } \\
\hline 4,0 & $-1,8595$ & 0,0886 & 14,74 & 14,98 & 15,37 & 16,09 & 17,02 & 18,12 & 19,31 & 20,14 & 20,74 \\
\hline 4,5 & $-1,8010$ & 0,0911 & 14,77 & 15,01 & 15,42 & 16,16 & 17,12 & 18,26 & 19,50 & 20,36 & 20,98 \\
\hline 5,0 & $-1,7504$ & 0,0937 & 14,79 & 15,05 & 15,46 & 16,23 & 17,23 & 18,41 & 19,69 & 20,58 & 21,22 \\
\hline 5,5 & $-1,7076$ & 0,0965 & 14,82 & 15,08 & 15,51 & 16,30 & 17,33 & 18,56 & 19,89 & 20,82 & 21,49 \\
\hline 6,0 & $-1,6716$ & 0,0994 & 14,85 & 15,12 & 15,56 & 16,39 & 17,45 & 18,72 & 20,11 & 21,08 & 21,78 \\
\hline 6,5 & $-1,6682$ & 0,1022 & 14,92 & 15,20 & 15,66 & 16,50 & 17,61 & 18,93 & 20,38 & 21,40 & 22,14 \\
\hline 7,0 & $-1,7061$ & 0,1053 & 15,06 & 15,35 & 15,82 & 16,70 & 17,84 & 19,23 & 20,76 & 21,85 & 22,64 \\
\hline 7,5 & $-1,7482$ & 0,1088 & 15,25 & 15,55 & 16,03 & 16,94 & 18,14 & 19,60 & 21,24 & 22,41 & 23,28 \\
\hline 8,0 & $-1,7681$ & 0,1125 & 15,46 & 15,77 & 16,27 & 17,22 & 18,48 & 20,02 & 21,77 & 23,04 & 23,99 \\
\hline 8,5 & $-1,7594$ & 0,1160 & 15,70 & 16,02 & 16,54 & 17,53 & 18,85 & 20,49 & 22,36 & 23,72 & 24,75 \\
\hline 9,0 & $-1,7148$ & 0,1189 & 15,95 & 16,28 & 16,83 & 17,86 & 19,25 & 20,97 & 22,93 & 24,38 & 25,47 \\
\hline 9,5 & $-1,6414$ & 0,1212 & 16,19 & 16,53 & 17,10 & 18,18 & 19,62 & 21,41 & 23,46 & 24,96 & 26,08 \\
\hline 10,0 & $-1,5409$ & 0,1229 & 16,42 & 16,78 & 17,37 & 18,49 & 19,99 & 21,84 & 23,93 & 25,47 & 26,61 \\
\hline 10,5 & $-1,4116$ & 0,1242 & 16,67 & 17,05 & 17,66 & 18,83 & 20,38 & 22,27 & 24,41 & 25,95 & 27,08 \\
\hline 11,0 & $-1,2589$ & 0,1247 & 16,96 & 17,35 & 18,00 & 19,21 & 20,81 & 22,74 & 24,89 & 26,42 & 27,53 \\
\hline 11,5 & $-1,0900$ & 0,1243 & 17,28 & 17,69 & 18,37 & 19,63 & 21,27 & 23,23 & 25,37 & 26,86 & 27,94 \\
\hline 12,0 & $-0,9385$ & 0,1232 & 17,65 & 18,08 & 18,78 & 20,08 & 21,76 & 23,74 & 25,85 & 27,31 & 28,34 \\
\hline 12,5 & $-0,8052$ & 0,1214 & 18,05 & 18,50 & 19,23 & 20,57 & 22,27 & 24,25 & 26,34 & 27,75 & 28,75 \\
\hline 13,0 & $-0,6971$ & 0,1192 & 18,48 & 18,94 & 19,68 & 21,05 & 22,77 & 24,75 & 26,81 & 28,18 & 29,14 \\
\hline 13,5 & $-0,6224$ & 0,1167 & 18,91 & 19,38 & 20,14 & 21,52 & 23,25 & 25,23 & 27,25 & 28,59 & 29,52 \\
\hline 14,0 & $-0,5705$ & 0,1140 & 19,35 & 19,82 & 20,59 & 21,98 & 23,71 & 25,67 & 27,66 & 28,97 & 29,88 \\
\hline
\end{tabular}




\section{DISCUSIÓN}

El presente estudio es el primero en proporcionar curvas de percentilos suavizadas mediante la aplicación del método LMS para la $\mathrm{CB}$ y las medidas derivadas de una muestra de más de 22000 niños y niñas procedentes de poblaciones de seis provincias de Argentina. Dado que estas medidas antropométricas son dependientes de la edad y del sexo, este estudio proporciona no solo los percentilos, sino también los valores lambda, mu y sigma, que permiten el cálculo de los puntajes z en ambos sexos y a cualquier edad, comprendida en el rango de 4 a 14 años.

La CB, así como el peso para la talla, han sido tradicionalmente utilizados para definir la desnutrición grave en los niños menores de 5 años de edad. Sin embargo, para la $\mathrm{CB}$, la $\mathrm{WHO}^{26}$ propone un único punto de corte independiente de la edad y del sexo y que corresponde a valores menores de $115 \mathrm{~mm}$. Esto puede conducir, de acuerdo con lo expresado por Abdel-Rahman

TABla 2. Percentilos $(P)$ y valores de la asimetría $(L)$ y coeficiente de variación $(S)$ para el área muscular en los varones y las mujeres

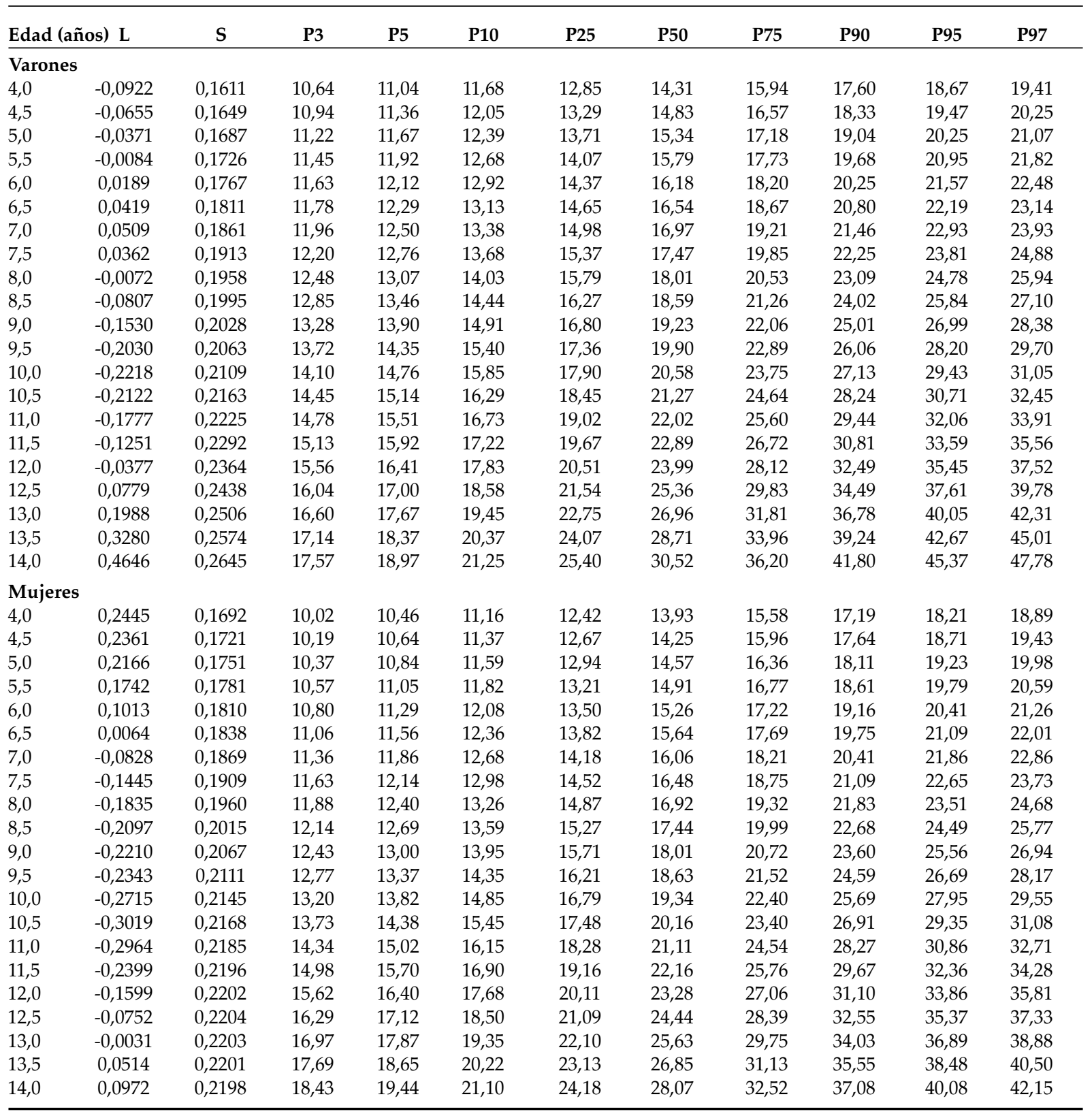


et al., ${ }^{27}$ al sobrediagnóstico o a la subestimación de la desnutrición aguda en el grupo de edad menor de 5 años, lo que reduce efectivamente la sensibilidad de esta medida. En tanto que otros autores proponen que un valor $<134 \mathrm{~mm}$ es indicio de riesgo y que los puntos de corte de 125 y 115 marcan el límite para la desnutrición moderada y grave, respectivamente. ${ }^{28}$ Es por ello por lo que el diagnóstico basado en la CB calculado con el método LMS, como lo realizado en este trabajo y los análogos de Addo et al. ${ }^{21}$ y Abdel-Rahman et al., ${ }^{27}$ sería más preciso al permitir definir nuevos puntos de corte específicos por sexo y edad.

Además, la fuerte correlación de la CB con diversos indicadores de adiposidad (peso e índice de masa corporal, porcentaje de grasa corporal y circunferencia de la cintura) permitiría también evaluar el sobrepeso y la obesidad de los niños de 9 a 11 años. ${ }^{10}$ Basándose en el análisis de curvas Receiver Operating Characteristic (ROC), Craig et al..$^{29}$ propusieron distintos puntos de corte de la

Tabla 3. Percentilos $(P)$ y valores de la asimetría $(L)$ y coeficiente de variación $(S)$ para el área grasa en los varones y las mujeres

\begin{tabular}{|c|c|c|c|c|c|c|c|c|c|c|c|}
\hline Edad (años) & $\mathbf{L}$ & $\mathbf{S}$ & P3 & P5 & P10 & P25 & P50 & P75 & P90 & P95 & P97 \\
\hline \multicolumn{12}{|l|}{ Varones } \\
\hline 4,0 & $-0,3827$ & 0,3057 & 4,91 & 5,21 & 5,72 & 6,74 & 8,20 & 10,11 & 12,41 & 14,15 & 15,45 \\
\hline 4,5 & $-0,3970$ & 0,3239 & 4,77 & 5,07 & 5,59 & 6,64 & 8,16 & 10,20 & 12,71 & 14,64 & 16,12 \\
\hline 5,0 & $-0,4107$ & 0,3429 & 4,62 & 4,92 & 5,45 & 6,53 & 8,11 & 10,29 & 13,03 & 15,19 & 16,86 \\
\hline 5,5 & $-0,4210$ & 0,3629 & 4,45 & 4,75 & 5,29 & 6,38 & 8,03 & 10,34 & 13,32 & 15,72 & 17,61 \\
\hline 6,0 & $-0,4234$ & 0,3835 & 4,24 & 4,54 & 5,08 & 6,19 & 7,87 & 10,30 & 13,51 & 16,15 & 18,28 \\
\hline 6,5 & $-0,4166$ & 0,4044 & 4,06 & 4,36 & 4,90 & 6,03 & 7,77 & 10,33 & 13,78 & 16,68 & 19,06 \\
\hline 7,0 & $-0,4023$ & 0,4250 & 3,96 & 4,27 & 4,82 & 5,99 & 7,83 & 10,56 & 14,33 & 17,55 & 20,21 \\
\hline 7,5 & $-0,3830$ & 0,4442 & 3,95 & 4,28 & 4,86 & 6,10 & 8,07 & 11,05 & 15,22 & 18,81 & 21,81 \\
\hline 8,0 & $-0,3576$ & 0,4614 & 4,02 & 4,37 & 4,99 & 6,33 & 8,47 & 11,74 & 16,35 & 20,36 & 23,72 \\
\hline 8,5 & $-0,3266$ & 0,4762 & 4,14 & 4,51 & 5,18 & 6,64 & 8,98 & 12,58 & 17,66 & 22,08 & 25,77 \\
\hline 9,0 & $-0,2937$ & 0,4888 & 4,28 & 4,69 & 5,42 & 7,01 & 9,58 & 13,52 & 19,07 & 23,88 & 27,87 \\
\hline 9,5 & $-0,2607$ & 0,4997 & 4,43 & 4,87 & 5,67 & 7,39 & 10,19 & 14,47 & 20,48 & 25,64 & 29,89 \\
\hline 10,0 & $-0,2268$ & 0,5086 & 4,57 & 5,04 & 5,90 & 7,76 & 10,77 & 15,38 & 21,78 & 27,23 & 31,69 \\
\hline 10,5 & $-0,1964$ & 0,5156 & 4,68 & 5,18 & 6,09 & 8,08 & 11,30 & 16,18 & 22,91 & 28,56 & 33,15 \\
\hline 11,0 & $-0,1759$ & 0,5208 & 4,76 & 5,29 & 6,25 & 8,34 & 11,71 & 16,81 & 23,79 & 29,62 & 34,30 \\
\hline 11,5 & $-0,1652$ & 0,5247 & 4,81 & 5,35 & 6,34 & 8,49 & 11,97 & 17,22 & 24,39 & 30,34 & 35,11 \\
\hline 12,0 & $-0,1641$ & 0,5276 & 4,83 & 5,38 & 6,38 & 8,56 & 12,09 & 17,44 & 24,73 & 30,79 & 35,65 \\
\hline 12,5 & $-0,1698$ & 0,5297 & 4,82 & 5,37 & 6,36 & 8,55 & 12,09 & 17,47 & 24,83 & 30,97 & 35,90 \\
\hline 13,0 & $-0,1799$ & 0,5308 & 4,78 & 5,33 & 6,31 & 8,48 & 11,99 & 17,35 & 24,74 & 30,93 & 35,94 \\
\hline 13,5 & $-0,1919$ & 0,5303 & 4,75 & 5,28 & 6,25 & 8,38 & 11,85 & 17,16 & 24,52 & 30,73 & 35,77 \\
\hline 14,0 & $-0,2043$ & 0,5287 & 4,72 & 5,24 & 6,19 & 8,29 & 11,70 & 16,94 & 24,25 & 30,44 & 35,49 \\
\hline \multicolumn{12}{|l|}{ Mujeres } \\
\hline 4,0 & $-0,2514$ & 0,3061 & 5,44 & 5,79 & 6,39 & 7,57 & 9,23 & 11,37 & 13,85 & 15,67 & 17,01 \\
\hline 4,5 & $-0,2332$ & 0,3223 & 5,24 & 5,60 & 6,21 & 7,42 & 9,14 & 11,39 & 14,01 & 15,95 & 17,39 \\
\hline 5,0 & $-0,2161$ & 0,3397 & 5,04 & 5,40 & 6,02 & 7,28 & 9,06 & 11,42 & 14,21 & 16,28 & 17,82 \\
\hline 5,5 & $-0,1979$ & 0,3581 & 4,83 & 5,20 & 5,84 & 7,13 & 8,99 & 11,47 & 14,43 & 16,65 & 18,31 \\
\hline 6,0 & $-0,1762$ & 0,3770 & 4,64 & 5,01 & 5,67 & 7,00 & 8,93 & 11,54 & 14,69 & 17,05 & 18,84 \\
\hline 6,5 & $-0,1560$ & 0,3962 & 4,47 & 4,86 & 5,53 & 6,91 & 8,94 & 11,70 & 15,05 & 17,59 & 19,51 \\
\hline 7,0 & $-0,1420$ & 0,4157 & 4,41 & 4,81 & 5,51 & 6,97 & 9,14 & 12,11 & 15,77 & 18,56 & 20,67 \\
\hline 7,5 & $-0,1303$ & 0,4347 & 4,43 & 4,86 & 5,61 & 7,18 & 9,53 & 12,80 & 16,86 & 19,98 & 22,36 \\
\hline 8,0 & $-0,1223$ & 0,4506 & 4,52 & 4,98 & 5,78 & 7,47 & 10,04 & 13,64 & 18,16 & 21,65 & 24,33 \\
\hline 8,5 & $-0,1240$ & 0,4608 & 4,69 & 5,18 & 6,04 & 7,86 & 10,65 & 14,59 & 19,58 & 23,47 & 26,46 \\
\hline 9,0 & $-0,1306$ & 0,4652 & 4,93 & 5,44 & 6,35 & 8,29 & 11,27 & 15,52 & 20,93 & 25,18 & 28,46 \\
\hline 9,5 & $-0,1313$ & 0,4663 & 5,14 & 5,68 & 6,64 & 8,67 & 11,79 & 16,26 & 21,97 & 26,46 & 29,94 \\
\hline 10,0 & $-0,1209$ & 0,4666 & 5,31 & 5,87 & 6,86 & 8,97 & 12,21 & 16,83 & 22,72 & 27,35 & 30,92 \\
\hline 10,5 & $-0,1020$ & 0,4675 & 5,45 & 6,02 & 7,06 & 9,24 & 12,60 & 17,36 & 23,40 & 28,11 & 31,73 \\
\hline 11,0 & $-0,0774$ & 0,4682 & 5,57 & 6,17 & 7,25 & 9,53 & 13,01 & 17,92 & 24,09 & 28,87 & 32,51 \\
\hline 11,5 & $-0,0480$ & 0,4677 & 5,70 & 6,34 & 7,46 & 9,84 & 13,46 & 18,51 & 24,79 & 29,59 & 33,23 \\
\hline 12,0 & $-0,0168$ & 0,4654 & 5,87 & 6,53 & 7,72 & 10,21 & 13,96 & 19,15 & 25,50 & 30,31 & 33,92 \\
\hline 12,5 & 0,0131 & 0,4612 & 6,07 & 6,77 & 8,02 & 10,62 & 14,52 & 19,83 & 26,26 & 31,05 & 34,62 \\
\hline 13,0 & 0,0388 & 0,4541 & 6,31 & 7,05 & 8,35 & 11,07 & 15,08 & 20,49 & 26,94 & 31,70 & 35,22 \\
\hline 13,5 & 0,0598 & 0,4444 & 6,59 & 7,36 & 8,71 & 11,51 & 15,60 & 21,06 & 27,46 & 32,14 & 35,57 \\
\hline 14,0 & 0,0757 & 0,4336 & 6,89 & 7,69 & 9,08 & 11,93 & 16,08 & 21,52 & 27,85 & 32,42 & 35,75 \\
\hline
\end{tabular}


Figura 1. Percentilos para circunferencia del brazo en los varones y las mujeres
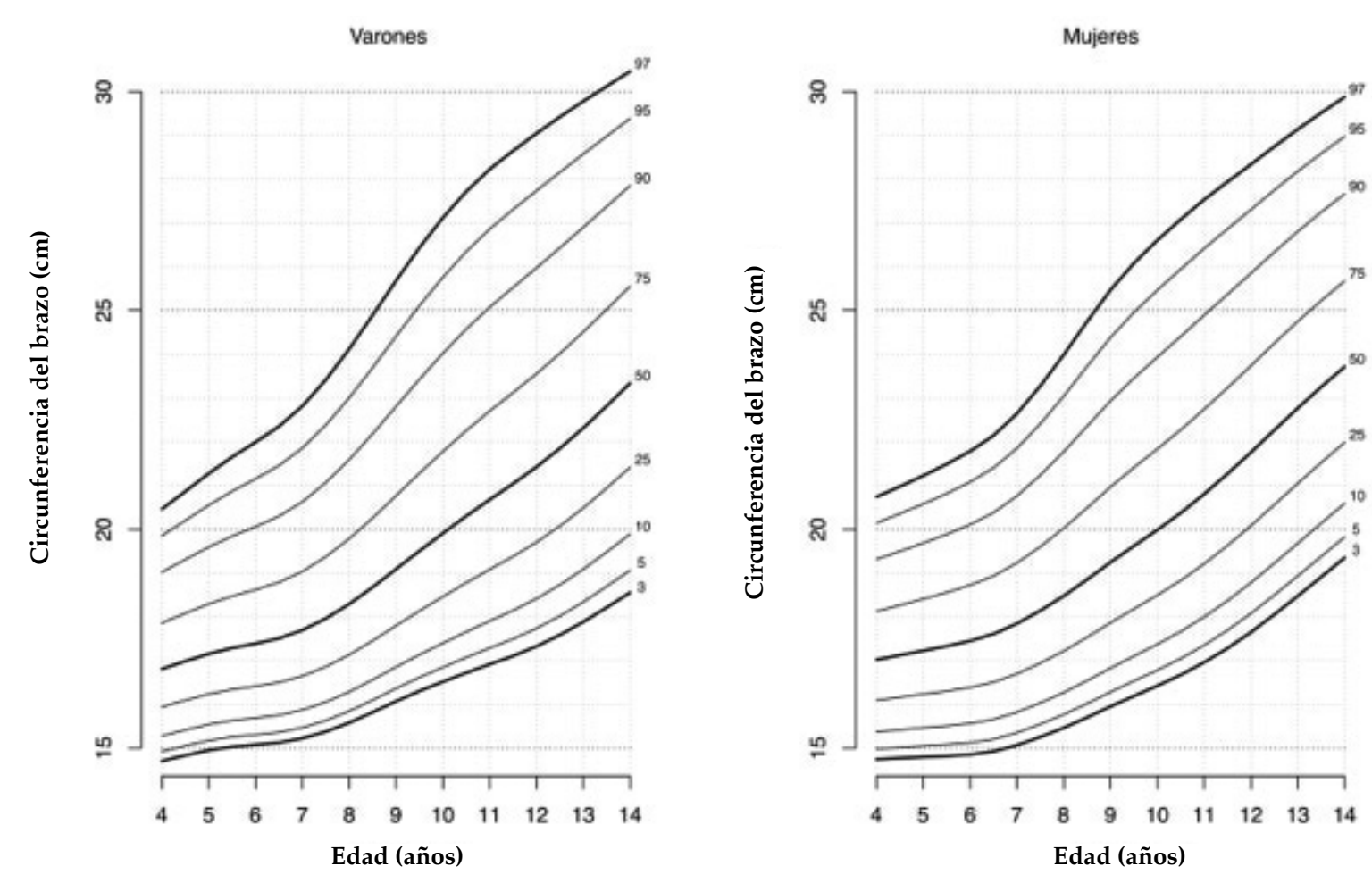

Figura 2. Percentilos para el área muscular en los varones y las mujeres
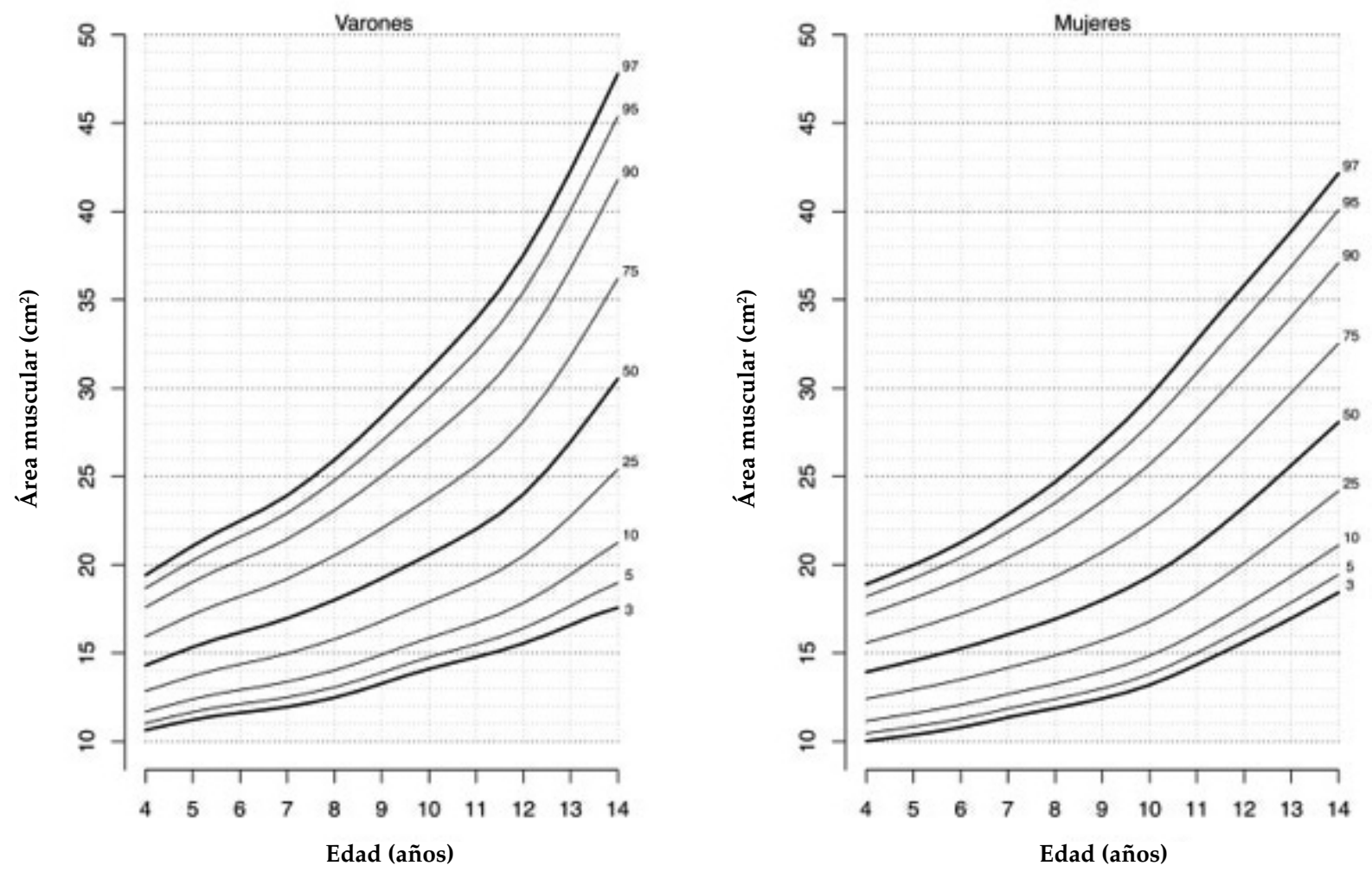
CB para identificar sobrepeso según sexo y edad. Sin embargo, los puntos de corte pueden variar en función del origen étnico de las poblaciones, ${ }^{10}$ razón por la cual, ante la inexistencia de un estándar, es recomendable contar con referencias locales.

En relación con lo anteriormente explicitado, la $C B$ de los niños analizados en el presente estudio fue, en general, mayor de lo reportado por Lejarraga et al. ${ }^{8}$ para los percentilos 50 y 97. Esta diferencia podría relacionarse con lo descrito por Guimarey et al., ${ }^{30}$ quienes informaron, transcurridos 30 años, incrementos significativos entre cohortes de niños de la ciudad de La Plata, Buenos Aires, del perímetro del brazo, así como del PT y del área adiposa mesobraquial.

Las diferencias observadas en el AG, a todas las edades, fueron a favor de las niñas, mientras que lo inverso ocurrió con el AM, lo que se correspondió, posiblemente, con la variación en la composición corporal propia de cada sexo. Sobre esta base, la suma de efectos tendió a compensarse y se expresaron en escasas diferencias sexuales en la CB. ${ }^{8}$

Dentro de las limitaciones de este trabajo, se puede considerar el hecho de que no se dispone de datos que abarquen todas las etapas del crecimiento. No obstante, posee, como principales fortalezas, que los datos antropométricos incluyen, en promedio, a más de 2000 escolares para cada una de las diez edades consideradas y que representan diferentes provincias con variabilidad ecogeográfica y socioeconómica. Se requiere, a futuro, la realización de estudios que permitan definir los puntos de corte para evaluar la malnutrición por defecto o por exceso y la validación de estos indicadores a través de su asociación con otros indicadores de adiposidad. En conclusión, los valores de percentilos, tabulados y graficados, de la circunferencia y de las AM y AG del brazo pueden ser considerados como una referencia local para estudios epidemiológicos y antropológicos.

\section{Agradecimientos}

Los autores agradecen a los niños y a sus padres por su colaboración desinteresada. A las autoridades escolares y a los maestros que facilitaron el trabajo de campo en los establecimientos educativos. A las instituciones que brindaron su apoyo financiero.

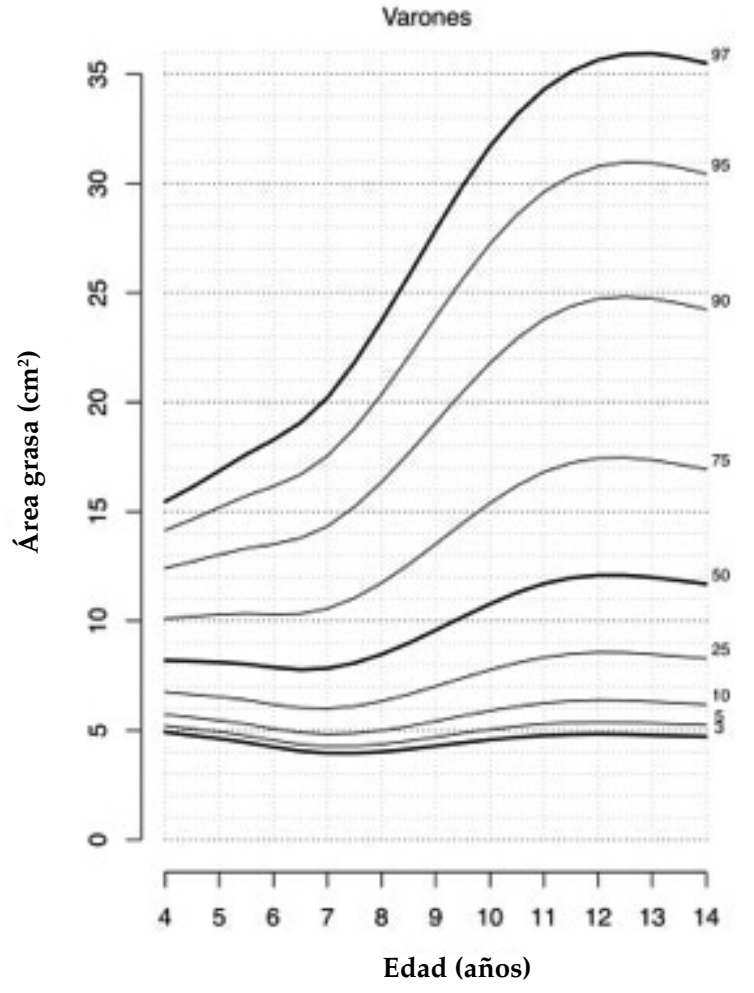

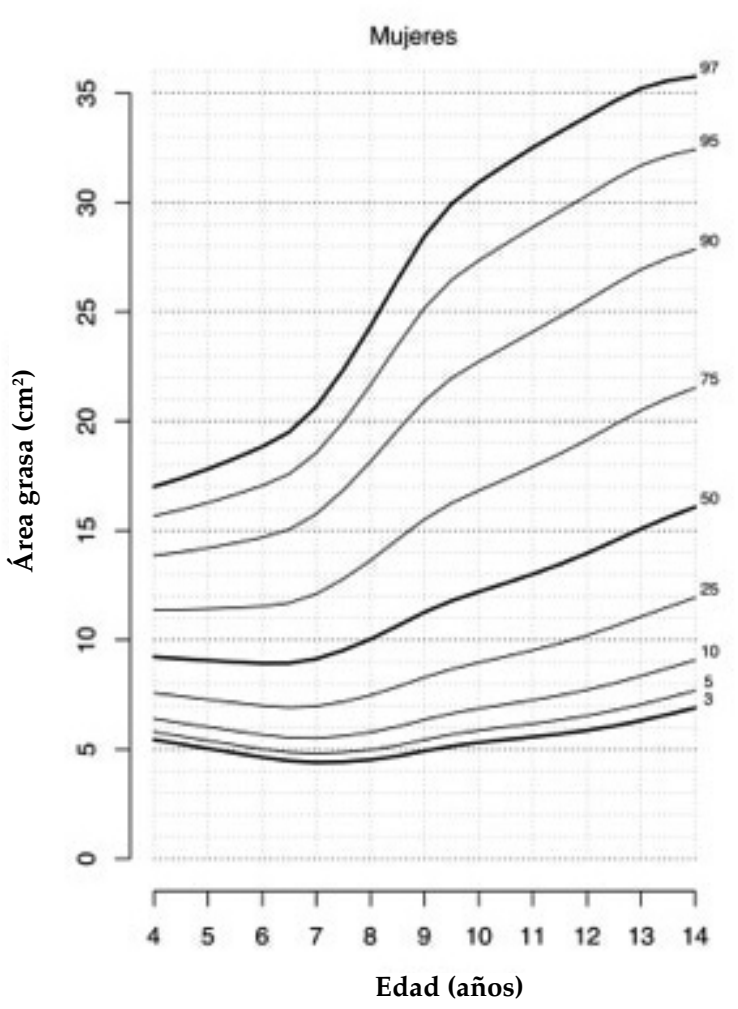




\section{REFERENCIAS}

1. Ugochukwu EG. Estimation of total muscle mass from simple anthropometric measurements for adults of Igbo ethnic group of South East Nigeria. Ann Bioanthropol. 2016;4(2):111-7.

2. Alfaro E, Bejarano I, Dipierri J, Quispe Y, et al. Percentilos de peso, talla e índice de masa corporal de escolares jujeños calculados por el método LMS. Arch Argent Pediatr. 2004;102(6):431-9.

3. Bolzán A, Mercer R. Seguridad alimentaria y retardo crónico del crecimiento en niños pobres del norte argentino. Arch Argent Pediatr. 2009;107(3):221-8.

4. Dahinten SL, Castro LE, Zavatti JR, Forte LM, et al. Growth of school children in different urban environments in Argentina. Ann Hum Biol. 2011;38(2):219-27.

5. Candelas N, Terán JM, López Barbancho D, Díaz MC, et al. Altitude effect on birth weight and prematurity in the Province of Catamarca (Argentina). Am J Hum Biol. 2015;27(4):526-9.

6. Pajuelo J, Amemiya I. Los indicadores antropométricos del brazo en los niños escolares del Perú. An Fac Med. 1998;59(1):23-28.

7. Jelliffe DB, Jelliffe EFP. The arm circumference as a public health index of protein-calorie malnutrition of early childhood. J Trop Pediatr (1967). 1969;15(4):253-60.

8. Lejarraga H, Markevich L, Sanchirico F, Cusminsky M. Reference tables of arm circumference from birth to 12 years of age for Argentinian girls and boys. Arch Latinoam Nutr. 1983;33(1):139-57.

9. Ayu DR, Aditiawati A, Anzar J, Bahar E. Upper arm circumference measurement for detecting overweight and obesity in children aged 6-7 years. Paediatr Indones. 2017;57(1):23-9.

10. Chaput JP, Katzmarzyk PT, Barnes JD, Fogelholm M, et al. Mid-upper arm circumference as a screening tool for identifying children with obesity: a 12-country study. Pediatr Obes. 2017;12(6):439-45.

11. Mantilla-Hernández MC, Niño-Bautista L, Prieto-Pinilla EE, Galvis-Padilla D, et al.. Validez de la cinta braquial para detección de desnutrición aguda en niñas y niños entre 6 y 59 meses de edad en escenarios de emergencias y desastres. Rev Salud Pública. 2014;16(2):195-207.

12. Behar AR. La construcción cultural del cuerpo: El paradigma de los trastornos de la conducta alimentaria. Rev Chil NeuroPsiquiatr. 2010;48(4):319-34.

13. Mramba L, Ngari M, Mwangome M, Muchai L, et al. A growth reference for mid upper arm circumference for age among school age children and adolescents, and validation for mortality: growth curve construction and longitudinal cohort study. BMJ. 2017;358:j3423.

14. Reilly JJ. Mid-upper arm circumference (MUAC): new applications for an old measure. Arch Dis Child. 2017;102(1):1-2.

15. Corvos Hidalgo CA. Evaluación antropométrica del estado nutricional empleando la circunferencia del brazo en estudiantes universitarios. Nutr Clín Diet Hosp. 2011;31(3):22-7.

16. Rolland-Cachera MF, Brambilla P, Manzoni P, Akrout $\mathrm{M}$, et al. Body composition assessed on the basis of arm circumference and triceps skinfold thickness: a new index validated in children by magnetic resonance imaging. $A m$ J Clin Nutr. 1997;65(6):1709-13.

17. McDowell MA, Fryar CD, Ogden CL, Flegal KM. Anthropometric reference data for children and adults:
United States, 2003-2006. Natl Health Stat Report. 2008;22(10):1-48.

18. World Health Organization. WHO Child Growth Standards. Length/ heigh-for-age, weight-for-age, weightfor-length, weight-for-height and body mass index-forage. Methods and development. NLM classification: WS 103. Geneva, Switzerland; 2006. [Consulta: 7 de marzo de 2018]. Disponible en: http:/ / www.who.int/ childgrowth / standards/Technical_report.pdf.

19. World HealthOrganization. WHOChildGrowthStandards: Head Circumference-for-Age, Arm Circumference-for-Age, TricepsSkinfold-for-Age and SubscapularSkinfold-for-Age Methods and Development. Geneva, Switzerland; 2007. [Consulta: 7 de marzo de 2018]. Disponible en: http:// www.who.int / childgrowth / standards / second_set / technical_report_2/en.

20. Frisancho AR. Anthropometric standards. An interactive nutritional reference of body size and body composition for children and adults. Ann Arbor: University of Michigan Press; 2008.

21. Addo OY, Himes JH, Zemel BS. Reference ranges for midupper arm circumference, upper arm muscle area, and upper arm fat area in US children and adolescents aged 1-20 y. Am J Clin Nutr. 2016;105(1):111-20.

22. Funes Lastra P, Agrelo F, Guita S, Chiquito FC de, et al. Estudio del crecimiento y desarrollo deniños normales de la ciudad de Córdoba a través de una muestra representativa. Córdoba: Ministerio de Bienestar Social, Departamento de Maternidad eInfancia, y Centro de Estudios de Crecimiento y Desarrollo del Niño; 1975.

23. Stewart A, MArfell-Jones M, International Society for the Advancement of Kinanthropometry. International Standards For Anthropometric Assessment. Glasgow, U.K: ISAK; 2011.

24. Cole TJ, Green PJ. Smoothing reference centile curves: the LMS method and penalized likelihood. Stat Med. 1992;11(10):1305-19.

25. Cole TJ. The British, American NCHS, and Dutch weight standard compared using the LMS method. Am J Hum Biol. 1989;1(4):397-408.

26. World Health Organization. WHOChild growth standards and the identification of severe acute malnutrition in infants and children: A Joint Statement by the World Health Organization and the United Nations Children's Fund. Geneva, Switzerland; 2009. Consulta: 12 de abril de 2018]. Disponible en: http://apps.who.int/iris/ bitstream / handle / 10665/44129/9789241598163_eng. pdf? sequence $=1$.

27. Abdel-Rahman SM, Bi C, Thaete K. Construction of Lambda, Mu, Sigma values for determining mid-upper arm circumference $Z$ scores in U.S. children aged 2 months through 18 years. Nutr Clin Pract. 2017;32(1):68-76.

28. Marrodán MD, Cabañas MD, Gómez A, González-Montero de Espinosa M, et al. Errores técnicos de medida en el diagnóstico de la desnutrición infantil: datos procedentes de intervenciones de Acción Contra el Hambre entre 2001 y 2010. Nutr Clín Diet Hosp. 2013;33(2):7-15.

29. Craig E, Bland R, Ndirangu J, Reilly JJ. Use of mid-upper arm circumference for determining overweight and overfatness in children and adolescents. Arch Dis Child. 2014;99(8):763-6.

30. Guimarey LM, Castro LE, Torres MF, Cesani MF, et al. Secular changes in body size and body composition in schoolchildren from La Plata city (Argentina). Anthrop Anz. 2014;71(3):287-301. 